\title{
Electrostatic interactions in a solution of linear micelles
}

\author{
Theo Odijk \\ Department of Polymer Technology, Faculty of Chemical Engineering and Materials Science, \\ Delft University of Technology, P.O. Box 5045, 2600 GA Delft, The Netherlands
}

(Received 9 March 1990; accepted 30 May 1990)

The interaction between charged linear micelles is taken into account via the first-order end contribution to the second virial coefficient. An asymptotic analysis of this term is given for finite lines in the Debye-Hückel approximation. The effective diameter introduced for infinite line charges also determines end effects to leading order. The size of linear micelles is a function of two ionic-strength dependent effects: one increasing with salt concentration, the other decreasing. There is a possibility of micellar growth with decreasing salt concentration at rather low ionic strength when the excluded-volume term starts to come into effect.

\section{INTRODUCTION}

Aggregating systems are very sensitive to molecular forces $^{1-3}$ and it is of fundamental interest to discover whether the interplay between interactions and aggregation can be given a statistical mechanical basis. Most of the previous theoretical work on linear micelles has treated these systems as ideal ${ }^{2,4,5}$ or the particles as hard bodies. ${ }^{6}$ There has also been a lot of discussion on the precise details of the molecular interactions (see, e.g., Refs. 1-3). However, there is one regime where it is reasonable to surmise that the intricate features of the forces should be irrelevant to a good approximation, namely when the ionic strength is low enough so that the Debye screening length becomes greater than the micellar diameter. In this limit the involved electrostatic interactions between nearest neighbor amphiphiles ought to have attained their maximum value. As the ionic strength is lowered even more, these local forces remain constant and only the long range electrostatic interactions increase. Several attempts at attacking this regime have appeared recently. ${ }^{7-10}$ This investigation focuses on the interaction between finite micelles in excess electrolyte.

Let us view a linear micelle as a finite line charge. The ionic cloud surrounding this polyion is virtually uniform except near the two end sections, each of which is approximately a Debye length $\kappa^{-1}$ long. Since an amphiphile close to the end interacts with fewer micellar charges than a surfactant situated in the midsection of the micelle, the electrostatic free energy is less than it would be if the ionic cloud were supposed to be completely uniform. Therefore, the end contribution to the free energy is negative and turns out to be proportional to the Debye length. Ultimately this implies that the micelle will grow as the ionic strength increases. A simple treatment of this effect is given in Ref. 8 .

The interaction of two finite line charges is more involved. Suppose the two micelles lie in a plane and the end of one micelle approaches the midsection of the other. The two particles will repel each other once the separation is less than about a Debye length. In this case the end contribution to the electrostatic free energy will be positive. If the micellar length $L$ is much greater than the Debye radius $\kappa^{-1}$, the end contribution to the second virial coefficient is of order $+L \kappa^{-2}$. Now suppose the micelles are not in a plane but cross. Then the end of one micelle will still be repelled by the midsection of the other. But this repulsion will be less than if the ionic clouds were supposed completely homogeneous; this nonuniformity has been discussed in the previous paragraph. A zero-order estimate of this end effect on the second virial coefficient would yield $-L \kappa^{-2}$, i.e., roughly speaking the magnitude of both end contributions is the same but they are of opposite sign. Accordingly, a fairly precise calculation of these two terms is of some importance. The object of this paper is to analyze the interaction between two finite line charges in the Debye-Hückel approximation and to discuss the implications for micellar growth.

\section{SECOND VIRIAL COEFFICIENT FOR FINITE POLYIONS}

The center of a micelle (1), modeled as a line charge, is the origin of our Cartesian coordinate system and the line itself defines the $z$ axis (see Fig. 1). Let $U=U(\mathbf{R}, \sin \gamma)$ be the electrostatic free energy when the center of a second line (2) is placed at $\mathbf{R}$ and the two lines are skewed at an angle $\gamma$. We let $\gamma$ be defined between 0 and $\pi$, and scale $U$ by $k_{B} T$ where $k_{B}$ is Boltzmann's constant and $T$ the temperature. The second virial coefficient $B_{2}$ is an orientational average of the first cluster integral $\beta_{1},{ }^{11}$

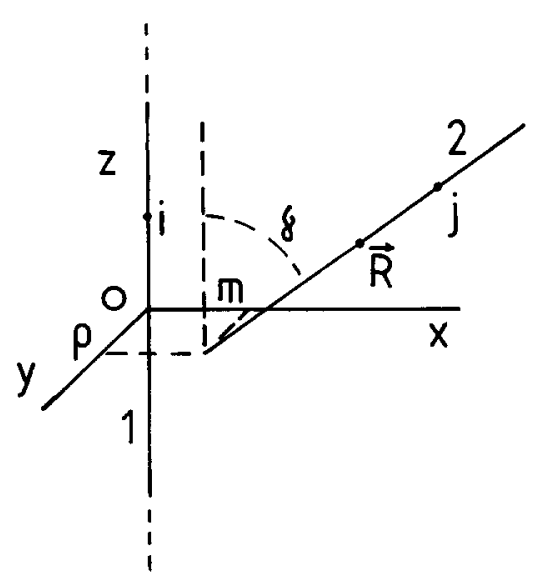

FIG. 1. Two interacting finite line charges. Line 2 is parallel to the $x z$ plane. 


$$
\begin{aligned}
& B_{2}=-\frac{1}{4} \int_{0}^{\pi} d \gamma \sin \gamma \beta_{1}(\gamma), \\
& -\beta_{1}(\gamma)=\int d \mathbf{R}\left(1-e^{-U}\right) .
\end{aligned}
$$

Usually $B_{2}$ is calculated by supposing that the energy $U$ arises from the interaction between two infinite line charges. Setting $U=U_{\infty}$ in this approximation we can write for $\beta_{1}(\gamma)$,

$$
-\beta_{1}^{0}(\gamma)=\int_{-(1 / 2) L \sin \gamma}^{(1 / 2) L \sin \gamma} d x \int_{-\infty}^{\infty} d y \int_{-(1 / 2) L}^{(1 / 2) L} d z\left(1-e^{-U_{\infty}}\right)
$$

This procedure is correct to order $L^{2} \kappa^{-1}$; for the calculation of $\beta_{1}^{0}$ (and $B_{2}^{0}$ ) see Refs. 12 and 13.

Our concern is the first-order correction to Eq. (3) which we can write as

$$
-\beta_{1}(\gamma)+\beta_{1}^{0}(\gamma)=4 L \kappa^{-2} I+O\left(\kappa^{-3}\right)
$$

when $L \kappa \gg 1$. First we note that there is no contribution of order $L \kappa^{-2}$ when the lines are almost parallel. This is because the integration in Eq. (1) would then be performed up to a maximum value of $\gamma$ of order $(\kappa L)^{-1}$. When $\gamma$ is larger than $(\kappa L)^{-1}$, there are four identical contributions in the integration of Eq. (2): there are four ends interacting with a midsection which itself in effect acts like an infinite line charge because $L \kappa \gg 1$. Each contribution involves the dimensionless integral $I$ which is calculated by placing a midsection along the $z$ axis as in Fig. 1 and analyzing its interaction with one end whose coordinates are specified by $m$ along the $x$ axis and $\rho$ parallel to the $y$ axis. The $z$ integration gives the factor $L$ and both $m$ and $\rho$ are scaled by the Debye length. Equations (2)-(4) imply

$$
\begin{aligned}
& I=I_{1}+I_{2}, \\
& I_{1} \equiv \int_{0}^{\infty} d m \int_{-\infty}^{\infty} d \rho\left(1-e^{-U}\right), \\
& I_{2} \equiv \int_{-\infty}^{0} d m \int_{-\infty}^{\infty} d \rho\left(e^{-U_{\infty}}-e^{-U}\right), \\
& U=U(m, \rho, \sin \gamma), \\
& U_{\infty}=U(-\infty, \rho, \sin \gamma), \quad m<0 \\
& \quad=0, \quad m>0 .
\end{aligned}
$$

Note that the contribution $I_{2}$ is negative since $U_{\infty}$ is larger than $U$.

In the Debye-Hückel approximation we have

$$
U=\frac{Q v^{2}}{\kappa} \int_{-\infty}^{\infty} d i \int_{0}^{\infty} d j \frac{e^{-r}}{r} \text {. }
$$

Here we have introduced the linear charge density $v$, i.e., the number of charges per unit length of micelle, and the Bjerrum length $Q=q^{2} / \epsilon k_{B} T$ with $q$ the elementary charge and $\epsilon$ the permittivity of the uniform solvent. In Fig. 1, $i$ is the distance of point $i$ from the origin, $j$ is the distance from the end of line 2 to point $j$, and $r$ is the distance between points $i$ and $j$. All lengths have been scaled by the Debye length.

$$
r^{2}=\rho^{2}+(i-j \cos \gamma)^{2}+(m+j \sin \gamma)^{2} .
$$

If we substitute $t=i-j \cos \gamma$ and $s=m+j \sin \gamma$, we can perform one integration ${ }^{14}$ in terms of a zero-order modified Bessel function of the second kind

$$
U=2 \pi^{-1} B \int_{m}^{\infty} d s K_{0}\left[\left(\rho^{2}+s^{2}\right)^{1 / 2}\right] .
$$

We have conveniently introduced two dimensionless parameters

$$
\begin{aligned}
& B \equiv A / \sin \gamma, \\
& A \equiv \pi Q v^{2} \kappa^{-1} .
\end{aligned}
$$

Equations (6), (7), and (10) are intractable as such so we first investigate the behavior of $U$ as a function of $m>0$ and $\rho$.

(1) For large $x, K_{0}$ is given by ${ }^{14}$

$$
K_{0}(x) \sim\left(\frac{\pi}{2 x}\right)^{1 / 2} e^{-x}
$$

It is straightforward to show that this represents an upper bound as well. So we have

$$
\begin{aligned}
& H(m, \rho) \equiv \int_{m}^{\infty} d s K_{0}\left[\left(\rho^{2}+s^{2}\right)^{1 / 2}\right]<\left(\frac{\pi}{2}\right)^{1 / 2} e^{-w} J(w, \rho) \\
& w \equiv\left(\rho^{2}+m^{2}\right)^{1 / 2} \\
& J(w, \rho) \equiv \int_{0}^{\infty} d u \frac{e^{-u}(w+u)^{1 / 2}}{(w+u+\rho)^{1 / 2}(w+u-\rho)^{1 / 2}} \\
& \simeq \pi^{1 / 2}\left(\frac{w+1}{\pi m^{2}+2 w+1}\right)^{1 / 2}
\end{aligned}
$$

For $w>1$,

$$
H(m, \rho) \sim\left(\frac{\pi}{2}\right)^{1 / 2} e^{-w} J(w, \rho) .
$$

(2) A lower bound is found by using one of the definitions ${ }^{14}$ of $K_{0}$,

$$
\begin{aligned}
K_{0}(x) & =e^{-x} \int_{0}^{\infty} d u \frac{e^{-x u}}{(2+u)^{1 / 2} u^{1 / 2}} \\
& >\left(\frac{\pi}{3}\right)^{1 / 2} \operatorname{erf} 1 e^{-x} x^{-1 / 2} \quad \text { for } x>1
\end{aligned}
$$

so that

$$
H>\left(\frac{\pi}{3}\right)^{1 / 2} \text { erf } 1 e^{-w} J(w, \rho) .
$$

(3) For small $\rho$ and $m$, a useful bound is

$$
H<\frac{1}{2} \pi \exp \left[-\left(\rho^{2}+m^{2}\right)^{1 / 2}\right]
$$

in view of

$$
\begin{aligned}
K_{0}\left[\left(\rho^{2}+s^{2}\right)^{1 / 2}\right] & =K_{0}\left[\left(\rho^{2}+m^{2}+2 m x+x^{2}\right)^{1 / 2}\right] \\
& <K_{0}\left[\left(\rho^{2}+m^{2}+x^{2}\right)^{1 / 2}\right],
\end{aligned}
$$

where we have used the substitution $s=m+x$.

(4) Finally, for $m=0$ the integration can be performed exactly, ${ }^{14}$

$$
H(0, \rho)=\frac{1}{2} \pi e^{-\rho} .
$$

All this analysis establishes that it is an excellent zeroorder ansatz to set

$$
\begin{aligned}
& U=B \exp \left[-\left(\rho^{2}+m^{2}\right)^{1 / 2}\right], \quad m>0, \\
& U=2 B \exp -(\rho)-B \exp \left[-\left(\rho^{2}+m^{2}\right)^{1 / 2}\right], \quad m \leqslant 0, \\
& U_{\infty}=2 B e^{-\rho} .
\end{aligned}
$$

Thus we perform an asymptotic expansion for large $B$ and 
estimate the corrections to $\mathrm{Eq} .(18)$ at the end of the analysis.

\section{A. Evaluation of $/ 1$}

We first use a well-known result obtained by an integration by parts which is valid for a function $f(\rho)$ monotone decreasing for $\rho>0$, with $f(\rho)=f(-\rho)$ and $f$ rapidly decaying at infinity

$$
\begin{gathered}
\int_{-\infty}^{\infty} d \rho\left(e^{-B f(\rho)}-1\right)=2 B \int^{f(0)} d g e^{-B g} f^{-1}(g), \\
f(\infty)=0 .
\end{gathered}
$$

The inverse $f^{-1}$ is defined by $\rho=f^{-1}(g)$, i.e., $g=f(\rho)$. Equations (8), (18), and (19) together with the substitution $u=B g$ yield

$$
I_{1}=2 \int_{0}^{\infty} d m \int_{0}^{B e^{-m}} d u e^{-u\left(\ln ^{2} \frac{B}{u}-m^{2}\right)^{1 / 2} .}
$$

The combination $B e^{-m}$ is awkward so it is eliminated by the substitution $y=B e^{-m}$,

$I_{1}=2 \int_{0}^{B} \frac{d y}{y} \int_{0}^{y} d u e^{-u}(\ln y-\ln u)^{1 / 2}\left(\ln \frac{B^{2}}{y}-\ln u\right)^{1 / 2}$.

This expression alludes to an expansion for large $\ln B$. However, one must proceed carefully since we have to contend with several logarithmic singularities. We must ensure that all expansions are uniformly convergent.

One potential nuisance occurs at $y=0$ so it is obviated by splitting up the $y$ integration

$$
I_{1}=I_{1 a}+I_{1 b}=\int_{0}^{1} d y+\int_{1}^{\infty} d y .
$$

We approximate the integral $I_{1 a}$ by substituting $u=y t$, noting that $\exp (-y t) \leqslant 1$ and then substituting $t=\exp -x$,

$$
I_{1 a}<2 \int_{0}^{1} d y \int_{0}^{\infty} d x e^{-x} x^{1 / 2}\left(2 \ln \frac{B}{y}+x\right)^{1 / 2} .
$$

An asymptotic expansion for large $\ln B / y$ is now possible. After the substitution $y=\exp -w$, another asymptotic expansion for large $\ln B$ yields

$$
I_{1 a}<(2 \pi)^{1 / 2} \ln ^{1 / 2} B+O\left(\ln ^{-1 / 2} B\right) .
$$

The other integral $I_{1 b}$ is now tackled readily:

$I_{1 b}=2 \int_{1}^{B} \frac{d y}{y} \int_{0}^{y} d u e^{-u}(\ln y-\ln u)^{1 / 2}\left(\ln \frac{B^{2}}{y}-\ln u\right)^{1 / 2}$

by noting the inequality

$$
\begin{aligned}
& (\ln y-\ln u)^{1 / 2}\left(\ln \frac{B^{2}}{y}-\ln u\right)^{1 / 2} \\
& \quad \leqslant \ln ^{1 / 2} y \ln ^{1 / 2} \frac{B^{2}}{y}+\frac{\ln ^{2} u-2 \ln B \ln u}{\ln ^{1 / 2} B^{2} / y \ln ^{1 / 2} y}
\end{aligned}
$$

An asymptotic analysis of Eq. (24) is then straightforward,

$$
I_{1 b}=\frac{\pi}{2} \ln ^{2} B+k_{1} \ln B-O\left(\ln ^{1 / 2} B\right),
$$

$k_{1}<2 \pi \gamma_{E} ; \gamma_{E}$ is Euler's constant.

\section{B. Evaluation of $/ 2$}

When $m$ is smaller than zero, we insert Eq. (18) into Eq. (7) and again use a form analogous to Eq. (19). On switching to the variable $n=-m$ we obtain

$$
I_{2}=2 B \int_{0}^{1} d g e^{-B g} \int_{0}^{\infty} d n[\rho(g, n)+\ln g-\ln 2] .
$$

We have replaced the limit $2-\exp -n$ by unity which introduces an error of order $B^{-1}$. This is much smaller than the terms retained. The function $\rho(g, n)$ is given implicitly by

$$
g=2 e^{-\rho}-e^{-\left(\rho^{2}+n^{2}\right)^{\prime / 2}}
$$

An integration by parts yields an explicitly known integrand. It is straightforward to show that $n(\rho+\ln g-\ln 2)$ tends to zero as $n$ becomes very large,

$$
I_{2}=-2 B \int_{0}^{1} d g e^{-B g} \int_{-\ln g}^{-\ln g+\ln 2} d \rho n .
$$

Next we introduce the variables $l=\rho+\ln g$ and $u=B g$,

$$
\begin{aligned}
I_{2}= & -2 \int_{0}^{\ln 2} d l \int_{0}^{B} d u e^{-u}\left[-\ln \left(2-e^{l}\right)\right]^{1 / 2} \\
& \times\left[2 \ln B-2 \ln u+2 l-\ln \left(2-e^{l}\right)\right]^{1 / 2} .
\end{aligned}
$$

With the help of the identity

$$
(E+F)^{1 / 2}=E^{1 / 2}+\frac{F}{E^{1 / 2}+(E+F)^{1 / 2}}
$$

one can establish

$$
I_{2}=-k_{3} \ln ^{1 / 2} B-O\left(\ln ^{-1 / 2} B\right),
$$

where

$$
k_{3}=2^{3 / 2} \int_{0}^{\infty} d z \frac{z^{1 / 2}}{\left(2 e^{z}-1\right)}
$$

and we note that all higher order terms converge.

\section{Analysis of correction terms}

\section{The case $m>0$}

In the leading integral $I_{1 b}=O\left(\ln ^{2} B\right)$ the main contribution comes from the region $y=O(B)$ and $u=O(1)$. Equations (19) and (20) then show that $m=O(\ln B)$ and $\rho=O(\ln B)$. From Eq. (15) we discern that on average $J$ $=O\left(\ln ^{-1 / 2} B\right)$. Nevertheless, the variables $m$ and $\rho$ often occur together in the combination $w=\left(\rho^{2}+m^{2}\right)^{1 / 2}$ $=O(\ln B)$, i.e., in the asymptotic evaluation there is a significant region where $\rho=O(\ln B)$ and $m=O(1)$. In that case Eq. (18) is highly accurate in view of Eq. (17). Thus a proper estimate for $J$ would be between $O(1)$ and $O\left(\ln ^{-1 / 2} B\right)$.

If we adopt the most pessimistic estimate for $J$, an improved iteration for $w$ is given by

$$
B e^{-w} J=O(1),
$$

i.e.,

$$
\begin{aligned}
& w=w_{0}-O\left(\ln \ln ^{1 / 2} B\right), \\
& w_{0}=O(\ln B) .
\end{aligned}
$$


Equations (23) and (25) then amount to

$$
I_{1}=\frac{\pi}{2} \ln ^{2} B+O(\ln B) .
$$

\section{The case $m<0$}

Here $\rho$ is again $O(\ln B)$ but it is not $\rho$ but $\Delta \rho$, the change in $\rho$, that is important. Equation (27) shows that $\Delta \rho=O(1)$. Moreover if we write $\left(\rho^{2}+m^{2}\right)^{1 / 2} \simeq \rho$ $+\frac{1}{2} m^{2} \rho^{-1}$ we expect $m^{2} \rho^{-1}=O(1)$ so that $m=O\left(\ln ^{1 / 2} B\right)$. This explains why $I_{2}=O\left(\ln ^{1 / 2} B\right)$. Hence $J$ is essentially $O(1)$ and $I_{2}$ is given by

$$
I_{2}=-k_{3} \ln ^{1 / 2} B+o(1) \text {. }
$$

\section{APPLICATION TO CHARGED MICELLES}

Inserting Eqs. (31) and (32) into Eqs. (1)-(7) and retaining leading terms we obtain the second virial coefficient for large $\ln A$,

$$
\begin{aligned}
B_{2} & =\frac{\pi}{4} L^{2} \kappa^{-1} \ln 2 A+\pi L \kappa^{-2} \ln ^{2} A+O\left(L \kappa^{-2} \ln A\right) \\
& \simeq \frac{\pi}{4} L^{2} D_{\text {eff }}+\pi L D_{\text {eff }}^{2} \\
& \equiv B_{2,0}+\left(\frac{4 D_{\text {eff }}}{L}\right) B_{2,0} .
\end{aligned}
$$

The first term is a classic result. ${ }^{11-13}$ The second term is new and its sign is positive. To render Eq. (33) more comprehensible we have introduced an effective diameter

$$
D_{\text {eff }} \equiv \kappa^{-1} \ln 2 A
$$

in analogy with the uncharged case: two uncharged rods of diameter $D$ would have a second virial coefficient $\frac{1}{4} \pi L^{2} D$. The effective diameter of charged rods at low ionic strength is significantly larger than the Debye radius. ${ }^{12,13}$ The second term in Eq. (33) which is proportional to $D_{\text {eff }}^{2}$ shows that the effective diameter $D_{\text {eff }}$ rather than the Debye radius $\kappa^{-1}$ is the basic scaling length for the interaction of a polyion end as it approaches the midsection of another macroion. When two polyions cross the end correction is negative but its absolute magnitude is proportional to $\kappa^{-3 / 2} D_{\text {eff }}^{1 / 2}$ [see Eq. (30)] so it is a higher order term.

It is tempting to generalize this result in two directions.

(1) The effective diameter $D_{\text {eff }}$ again determines positive end corrections for the higher virials and some combination of $D_{\text {eff }}$ and $\kappa^{-1}$ pertains to negative end effects. On the whole, one expects the positive contributions to dominate. For example, we could write for the third virial coefficient

$$
B_{3} \simeq\left(\frac{4 D_{\text {eff }}}{L}\right) B_{2,0}^{2}+k_{4}\left(\frac{D_{\text {eff }}}{L}\right)^{2} B_{2,0}^{2},
$$

where $k_{4}$ is positive and the first term was proposed in Ref. 15.

(2) If the micelles have a nonzero diameter and the charge density is high so that the Debye-Hückel approximation no longer holds, $D_{\text {eff }}$ is given by the procedure outlined in Ref. 16 for infinite cylindrical polyelectrolytes. First, as pointed out in Ref. 8, for highly charged polyions the effective charge density is quite uniform even near the ends. This assertion follows from detailed calculations on very short rods by Ramanathan and Woodbury. ${ }^{17}$ Secondly, the outer region of the double layer is important so the procedure outlined in Ref. 16 remains intact. Furthermore, as $D_{\text {eff }}$ tends to $D$, the intrinsic diameter at very low charge density, Eq. (33) would reduce to the Onsager result for cylinders with spherical caps ${ }^{11}$

$$
B_{2}=\frac{\pi}{4} L^{2} D+\pi L D^{2}+O\left(D^{3}\right) .
$$

We are now in a position to postulate an expression for the chemical potential $\mu$ of an amphiphile in a micelle which we constrain to be monodisperse for simplicity

$$
\begin{aligned}
s \mu= & \text { extensive terms }+K+\ln \varphi-n \ln s \\
& -\frac{A}{\pi}+h\left(\frac{\varphi D_{\text {eff }}^{2}}{D^{2}}\right) .
\end{aligned}
$$

The unspecified extensive terms are proportional to $s$, the number of surfactants within one micelle. The next three terms come from the current theory of uncharged micelles. $^{2,4.5}$ The cap contribution $K$ is deemed to be independent of the ionic strength ${ }^{8}$ when $\kappa D<1$. The volume fraction $\varphi=\frac{1}{4} \pi L D^{2} \rho_{m}$ is linear in the number density $\rho_{m}$ of micelles. The number $n$ pertains to the number of degrees of freedom of every micelle. In Ref. 5 it was argued that $n$ should equal 6 . The term proportional to $A$ was introduced in Ref. 8 and relates to the nonuniformity of the double layer of a single micelle at its ends. It bears a negative sign since a charge at one end experiences fewer interactions than charges in the midsection. Note that if the charge density is high we may have to use an effective one pertaining to the inner double layer. ${ }^{8}$

The function $h$ has the following properties:

(1) For small $x$,

$$
h(x)=8 x+O\left(x^{2}\right)
$$

so that it conforms to the end term given by Eq. (33),

(2) $h^{\prime}(x)>0$.

This indicates that the end contribution to the electrostatic interaction increases as the end of one micelle gets close to a cluster of other micelles. This has been strictly proved here for binary collisions. It seems justifiable for the higher order virial terms since the effective diameter is the relevant electrostatic scale as a charged rod nears a cluster. Negative end terms are connected with a smaller scale, in between the Debye length and the effective diameter.

Minimization of $\mu$ with respect to $s$ gives the optimum number of amphiphiles within one micelle,

$$
n \ln s_{m}=K+\ln \varphi+n-\frac{A}{\pi}+h\left(\frac{\varphi D_{\mathrm{eff}}^{2}}{D^{2}}\right) .
$$

As the 1-1 electrolyte concentration $n_{s}$ decreases the micelle will first diminish in size because the $A$ term dominates [see Eq. (11); the Debye length is given by $\left.\kappa^{2}=8 \pi Q n_{s}\right]$. Nevertheless, when the ionic strength gets low enough, the excluded volume $h$ comes into play. Since it increases more rapidly than the $A$ term decreases, one expects the micelles to begin expanding again. A numerical example, will bear this out. Let the micelles have a charge density $v \simeq Q^{-1}$ so that the Debye-Hückel approximation is applicable. At reasona- 
bly low volume fractions, the terms in Eq. (37) depending on the ionic strength can then be written as

$$
\frac{8 \varphi \ln ^{2}(\pi / Q \kappa)}{D^{2} \kappa^{2}}-\frac{1}{\kappa Q} .
$$

When the micelles are dissolved in water at $25^{\circ} \mathrm{C}$, the Bjerrum length $Q$ equals $0.71 \mathrm{~nm}$. If their diameter $D$ is $4 \mathrm{~nm}$ and the micellar volume fraction $\varphi$ is 0.02 , the micelles attain their minimum size when $\kappa^{-1}=5.3 \mathrm{~nm}$, i.e., at an ionic strength of $0.0032 \mathrm{M}$. The effective diameter is then $D_{\text {eff }} \simeq 17$ nm so that the effective volume fraction $\varphi D_{\mathrm{eff}}^{2} / D^{2}$ is about 0.35 . Thus, we are pushing the theory close to its limits of validity (with regard to higher order virial terms, excess salt condition, the solution not becoming liquid crystalline). Note that the growth constant $K$ must be large enough so that the micelles can be modeled as slender rods under all circumstances.

\section{CONCLUDING REMARKS}

We have put forth asymptotic arguments proving that the end contribution to the second virial coefficient is positive and to leading order determined by an effective electrostatic diameter. Ultimately we have to add to the expression for the size of a linear micelle a term increasing strongly with decreasing ionic strength. Under the right circumstances this excluded-volume effect could offset a term introduced previously ${ }^{8}$ to explain micellar growth with increasing salt concentration. We raise the possibility of the micelles grow- ing again with diminishing salt concentration at rather low ionic strength.

'J. N. Israelachvili, Intermolecular and Surface Forces (Academic, London, 1985).

JJ. N. Israelachvili, D. J. Mitchell, and B. W. Ninham, J. Chem. Soc. Faraday Trans. 272, 1525 (1976).

${ }^{3}$ H. Wennerström and B. Lindman, Phys. Rep. 52, 1 (1979).

${ }^{4}$ R. Nagarajan and E. Ruckenstein, J. Colloid Interface Sci. 71, 580 (1979).

${ }^{5}$ W. E. McMullen III, W. M. Gelbart, and A. Ben-Shaul, J. Phys. Chem. 88, 6649 (1984).

${ }^{6}$ W. M. Gelbart, W. E. McMullen III, and A. Ben-Shaul, Mol. Cryst. Liq. Cryst. 132, 325 (1986).

${ }^{7}$ W. E. McMullen, W. M. Gelbart, and Y. Rosenfeld, J. Chem. Phys. 85, 1088 (1986). This work treats the isotropic-nematic transition without taking account of the screening from small ions. In principle the excesssalt version of this approach would contain our virial terms. Still, it is unclear at present how well the approximations of "Gaussian lines" and "optimized charge smearing" would be able to reproduce our logarithmic terms [Eq. (33]. These do require a precise analysis.

${ }^{8}$ T. Odijk, J. Phys. Chem. 93, 3888 (1989).

${ }^{9}$ S. A. Safran, P. A. Pincus, M. E. Cates, and F. C. MacKintosh, J. Phys. (Paris) 51, 503 (1990).

${ }^{16}$ F. C. MacKintosh, S. A. Safran, and P. A. Pincus (preprint).

"L. Onsager, Ann. N.Y. Acad. Sci. 51, 627 (1949).

${ }^{12}$ D. Stigter, Biopolymers 16, 1435 (1977).

${ }^{13}$ M. Fixman and J. Skolnick, Macromolecules 11, 863 (1978).

${ }^{14}$ I. S. Gradshteyn and I. M. Ryzhik, Table of Integrals, Series and Products (Academic, Orlando, 1980).

${ }^{15}$ T. Odijk, Macromolecules 19, 2313 (1986).

${ }^{16} \mathrm{~A}$. Stroobants, H. N. W. Lekkerkerker, and T. Odijk, Macromolecules 19, 2232 (1986).

${ }^{17}$ G. V. Ramanathan and C. P. Woodbury, J. Chem. Phys. 77, 4133 (1982). 